\title{
Digital transformation of logistic processes for a consumer goods company based on RF tags
}

\author{
Oksana Yu. Iliashenko \\ Higher School of Business and \\ Management \\ Institute of Industrial Management, \\ Economics and Trade \\ Peter the Great Saint Petersburg \\ Polytechnic University \\ Saint Petersburg, Russia \\ ioy120878@gmail.com
}

\author{
Grigorii M. Shestakov \\ Higher School of Business and \\ Management \\ Institute of Industrial Management, \\ Economics and Trade \\ Peter the Great Saint Petersburg \\ Polytechnic University \\ Saint Petersburg, Russia \\ gregshestakov@gmail.com
}

\author{
Victoria M. Iliashenko \\ Higher School of Business and \\ Management \\ Institute of Industrial Management \\ Economics and Trade \\ Peter the Great Saint Petersburg \\ Polytechnic University \\ Saint Petersburg, Russia \\ vmi1206@yandex.ru
}

\begin{abstract}
Logistics is one of the most important processes to be automated for a company producing consumer goods. With the using of modern technologies, such as RF tags, you can not only fully automate the process of receiving and delivering products, but also create a platform for digital transformation to an autonomous warehouse. This paper will consider the possibility and necessary conditions for the transition of warehouse logistics to the use of RFID technologies.
\end{abstract}

Keywords-digital transformation, warehousing logistic, $R F$ tags

\section{INTRODUCTION}

A. Analysis of the current approach to the warehouse logistics organization for the FMCG companies

Consider the process of movement of resources within the production of everyday goods. Two types of resources are coming on production: components and semi-finished products. Components - chemical reagents necessary for the production of goods. Semi-finished products - corrugated boxes, containers, etc., necessary for packaging products. All deliveries must be accepted, entered into the ERP system and pasted the sticker of the ERP system. Then each delivery is determined at its warehouse, separately for chemistry, separately for semi-finished products.

Then comes the production process, which in turn is divided into 2 subprocesses: mixing and packing. Mixing is the process of creating a product; packaging is the packaging of a product in a container for shipment to the final consumer. The mixing process is as follows: components enter the digester, with some of the components occupying the greatest mass in the upcoming cooking (for example, water) coming automatically, some of the components are manually loaded by the cooking operator. The manually loaded components are taken by the weight operators in the "supermarket". It is a small warehouse that is located directly in the mixing shop. In the same workshop, the weight operators measure the required number of components according to the flow chart and leave them in the cart, which is then taken by the cooking operator and adds all the components to the digester. From the digester, the finished product enters the packaging workshop, where it is packed in semi-finished products, packed and sent to a shipping warehouse. In terms of logistics, this process has the following steps:
- acceptance of components;

- delivery of components to warehouses;

- delivery of chemistry to the supermarket;

- collecting the components necessary for mixing the batch of the product;

- delivery of semi-finished products to the filling line;

- delivery of finished products to the shipping warehouse;

- shipment of finished products.

However, any delay in performing each of these stages leads to a halt in the entire production process, which may result in penalties on the part of customers. Considering the constantly changing requirements for supplies, automation of the logistics process is vital for maintaining the competitiveness of production

Let us consider in more detail the actions of the logistics department employees at each of the arising considered stages.

- Acceptance of components. The employee needs to check whether all the components are in place according to the documents, then add the components to the ERP system and stick stickers with a bar code on the pallet, with the help of a bar code, this delivery will be monitored in the future.

- Delivery of components to warehouses. The arriving components are determined to the appropriate warehouse, in this case the location is selected based on whether there is another component in the cell or not. To register a component in a cell, an employee needs to scan the delivery barcode and the barcode of the cell.

- Delivery of chemistry to the supermarket. At the beginning of the working day, the logistics officer collects the necessary components according to the current production plan, and since the components are delivered in whole pallets, the employee picks up only part of the components in individual packaging and takes them to the supermarket, then the employee needs to print an individual bar code for packaging, 
scan it, and then scan the barcode of the cell in which the component will be placed.

- Collection of components required for mixing batch of the product. At this stage, the weight operator collects all the necessary components for cooking. To do this, he takes a trolley with a barcode, drives up to the desired cell, scans the barcode of the cell, then scans the barcode of the required component, then scans the barcode of the cell. If the quantity of any component is not a multiple of the packing volume (for example, 30 $\mathrm{kg}$ is required, and packs are supplied $25 \mathrm{~kg}$ each), the remainder should be additionally hung. To do this, the weight operator is sent to the weighing unit, it scans the barcode of the cart, the barcode of the component to be hung, and the barcode of the cart. Then, it measures the required amount of the component and prints a new bar code for the remainder, in the future the system will know that there is already some amount of this component in the weighing and will direct the operator to the weighing, and not to the supermarket. After weighing, the operator takes the cart to the parking area of finished components, scans the barcode of the cart and the barcode of the zone.

- Delivery of semi-finished products to the filling line. The logistics employee, according to the current plan, delivers semi-finished products to the line, at the time of the start of packaging. To do this, you need to scan the barcode of the ERP system and enter into it information that the delivery went to the packaging department. It is important to understand that the employees delivering components and semi-finished products are different, since these resources are located in different warehouses.

- Delivery of finished products to the shipping warehouse. From the line, finished products are conveyed to a warehouse by conveyor, where they pack and glue a new bar code - the finished products.

- Shipment of finished products. Next, the finished products are shipped from the warehouse, and the necessary documents are filled in on it.

\section{B. Identification of current problems of the approach to warehouse logistics based on bar-coding}

Despite the fact that bar coding is quite a powerful tool for warehouse automation, let's consider what problems may arise when using such a systematic approach. The main function of the barcode is information storage. To read the information, you need special technical tools and direct bar code visibility. It is also difficult to automatically read the barcode without help, and the barcode stickers themselves are often subject to deformation, as a result of which the information becomes unreadable. Based on this, the following problems can be identified:

- Great influence of the human factor. All information that the information system receives will depend only on the employee. If there is any error on the part of the operator, there may be collisions in the system that need to be fixed.

- For complete information, you must record any action. Because of this, the number of actions that the operator must perform significantly increases. For example, instead of one action "transfer packing to cart", 4 actions are performed: "scan cell barcode" - "scan packing barcode" - "slide packing into cart" - "scan basket with barcode". Thus, firstly, the time required to transport the necessary components increases significantly; secondly, the employee appears a lot of routine actions that do not increase the value of the final product.

- Low information security. In a production environment, a barcode sticker is very easy to damage, so another action appears - "printing a new barcode". An operator error is also possible, which will cause the sticker to be reprinted.

- Instant data reading. The system receives information about the status of the bar code only at the moment when it is scanned, whereas for the system it looks like an instantaneous movement from point A to point B. Thus, there is no possibility of obtaining information about exactly how this path was taken.

Thus, despite the objective advantages of using a barcoding system, it has drawbacks that can and should be disposed of. In addition, an alternative system is needed to switch to a fully autonomous warehouse, since it is extremely difficult for automatic machines to work with bar codes. One such alternative is to use a system based on RF tags.

\section{METHODS}

\section{A. Analysis.}

The current processes occurring in warehouse logistics are analyzed, the processes involved in the value chain are identified. The market analysis in the field of RFID was carried out, and differences from existing bar-coding systems were revealed. Then NVAA analysis was carried out, which revealed many actions that did not add value to the final product in the process of delivering resources to production [1]

\section{B. Modeling.}

The main method used in the automation of the company's logistics processes is the ADM method of TOGAF architectural approach [2], which application for different industries are actively developed in Peter the Great Saint Petersburg Polytechnic University [3,4,5,6]. Using this method, a model of a business layer, an application layer, and a technology layer was compiled from the point of view of the logistic processes implementation.

\section{LITERATURE REVIEW}

The use of RF tags in logistics is one of the main trends of the last decade. Thanks to technological progress, each year the tags become more accurate, smaller, and production is cheaper. It is enough to compare the reviews of these technologies over time, for example, in 2006 [7] and in 2016 [8] to see how much RF tag technology has developed over such a period. Positive dynamics is observed in market research RF tags [9]. Thus, it can be argued that the use of RFID technology - the transition to a qualitatively new level of automation, including in logistics. For a full analysis, it is also necessary to understand what processes exist in production [10] and including what features the FMCG 
manufacturing company has [11]. Also important is the knowledge of logistic processes, including processes in warehouse logistics [12,13,14], as well as an understanding of the modern bar-coding systems principles [15]

\section{RESULTS}

\section{A. The possibilities of implementing RFID in warehouse logistics}

RFID (Radio Frequency Identification) is a method for automatic identification of objects, in which data stored in RF tags are read or written using radio signals. The system requires two components: a tag on which information will be recorded, and a reader that reads information and writes data on the tag. In this case, the tags can be active and passive. The active tag has its own power source and can read and transmit information from sensors. Unlike barcodes, RF tags are extremely difficult to create under production conditions. Accordingly, tags will have to be purchased, but because of the possibility of overwriting the data on the RF tag, they can be reused. There are already prototypes of technologies that allow making the production of RF tags autonomous, but so far such technologies are still at the testing stage.

Thus, the following interaction scheme in warehouse is possible. Each cell in the warehouse has an active label that, in addition to information about the component stored in it, transmits information about temperature, vibration, etc. Also, each cart and a pallet with components has a label. The label needs to be pasted only on the pallet. Then the system can automatically determine which cell the component is in (by calculating a label adjacent to the component) and determine the components in the empty cells. Since readers have the ability to read up to 1000 tags, the required number will remain within reasonable limits [16].

In order to deliver the component packaging to the supermarket, it is necessary to place a new label on it, transport it and put it in an empty cell. The system will automatically determine the new packing position. For the weight operator, it is also necessary to put the necessary components in the cart and transport them by weight. Information system can be connected to the weights of the weight. Thus, the system will automatically find out how much component is left in the package. Due to the fact that most modern ERP systems have an API for working with RFID, data registration will also be automatic [17].

To transport semi-finished products, there will also be no need to read bar codes, and when integrating the RFID system with production line sensors, it will be possible to calculate the remaining number of semi-finished products and promptly bring them up.

For finished products, RF tags are no longer needed, since we assume that customers will use a bar-coding system in the future, so the system should be encapsulated. With the global transition of all participants in the supply chain to RFID, the final barcode is replaced with an RF tag.

\section{B. Prerequisites for RFID implementation}

The main problem in the implementation of RFID sufficient maturity of the complex business processes in production. By itself, an RFID-based system without additional support for existing information systems would be a waste of budget. Inside the sequence "manual labor - partial automation - full automation", RFID is a means to move from partial automation to full automation, and for maximum efficiency it requires compliance with a number of conditions, such as [18]:

- Availability of a well-functioning ERP or similar system with RFID integration. The ERP system only collects and stores data. To analyze and visualize this data, it is necessary to transfer it to another system that has the corresponding functionality.

- Clearly written job descriptions for each production employee. This requirement arises at the stage of introducing the bar-coding system, however, if using bar-coding a barcode can be printed by an employee whose job it is not included, this is not possible in the RFID system, respectively, certain employees should be in charge of arranging these tags.

- Ability to cover a large area of production with wireless networks. Readers need to transfer information to work. The most reasonable thing to do is using wireless networks. Consequently, the area in which the reader is located must be covered by wireless networks. It is fair to say that in order to use a barcoding system, scanners also need wireless networks for transmitting data to the primary site, but unlike the RFID system, the entire work area must be covered by wireless networks for the stable operation of scanners.

- Competently written formed technical task. One of the main conditions for the implementation of any system is the availability of a well-formed full-fledged technical specification. For an RFID system, this requirement is critically important, since unlike the bar-coding system, there will be insufficiently prescribed functionality and system requirements. It will also be necessary to clearly describe the algorithm of the system, methods of coding information, requirements for additional software and conditions for integration with the existing information system.

- Availability of MES system. An optional, but desirable condition. To fully unlock the potential of an RFID system, many sensors are needed, which will automate more processes. If you have an MES system, no additional installation of such sensors is required; it will be enough to integrate them into the RFID system. If there is no MES system, then measures will also be needed to install and configure sensors on production lines.

\section{The process of implementing RFID in production}

For an RFID system implementation project, as well as for any project, it is necessary to create a project team. The main objectives of the implementation of RFID technology will be [19]:

- integration of RFID with the existing information system;

- arrangements for install the necessary sensors; 
- training personnel to work with the new system;

- arrangements for installing readers, order RF tags.

\section{The results of using RFID technology in logistics} processes

Let's consider the organization of logistics processes without using RFID and using RFID technology. We construct a model of business processes using the TOGAF standard (Fig. 1).

This model was built only taking into account the activities of the logistics department, the other actors were not considered for the convenience of perception of the model. Next, decompose the process of "Raw materials delivery to production" (Fig. 2).

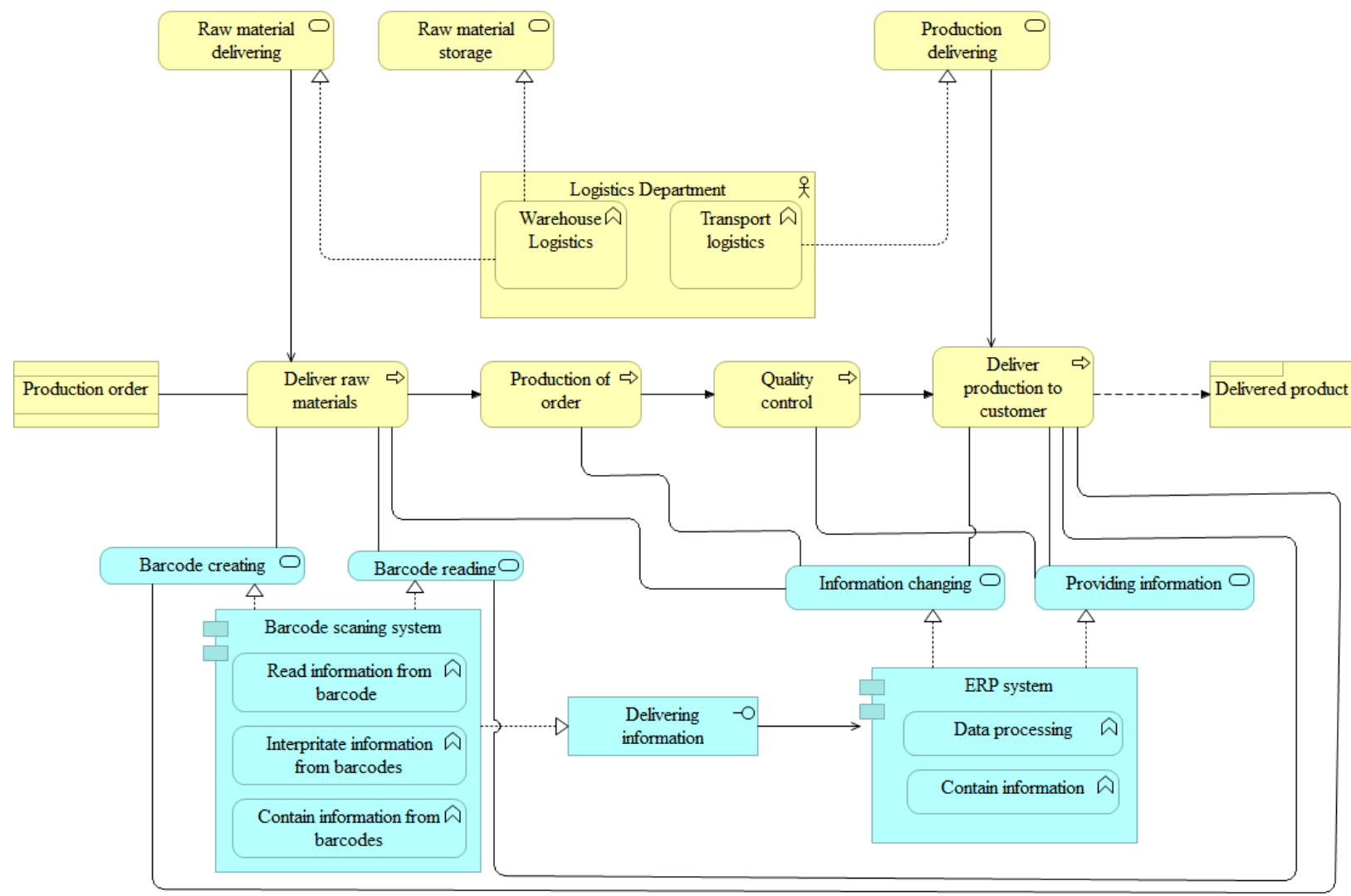

Figure 1 Producing order process as is

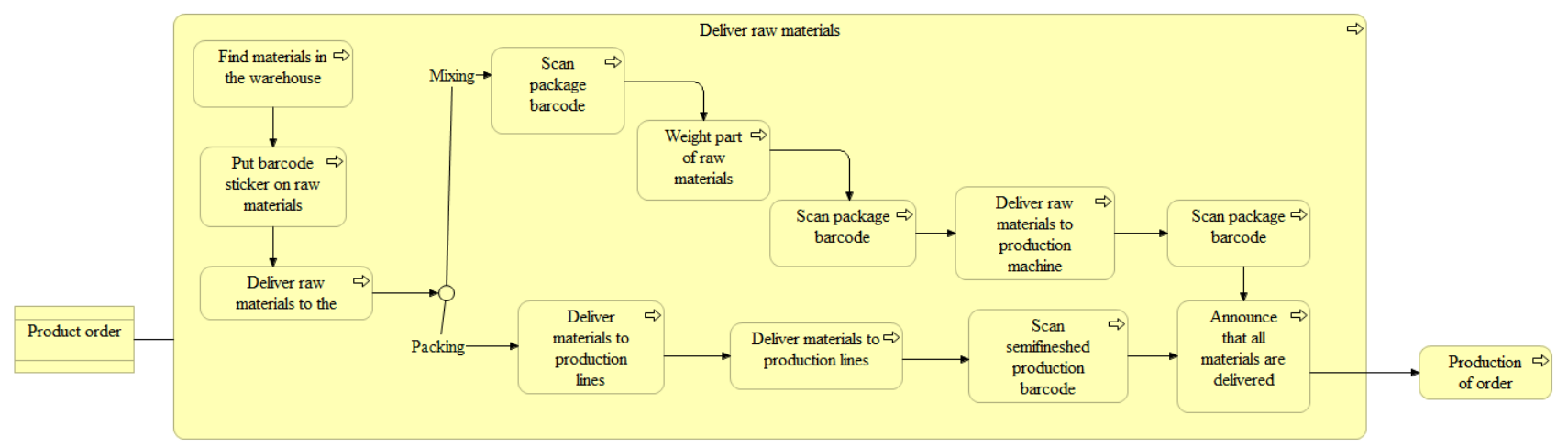

Figure 2 Decomposition of deliver raw materials process as is

The services of the application layer used in this process were not considered for the convenience of perception of the scheme. You may notice that with the existing system there are a large number of actions "scan barcode". In order to understand why this happens, consider the application layer (Fig. 3).

As can be seen from the diagram, the process launch starts with the event "barcode scanning", initiated by the employee. 
The process cannot be started without human intervention. Now consider the scheme after the introduction of RFID technology (Fig. 4).
We decompose the process of "delivering raw materials to production" using RF tags (Fig.5)

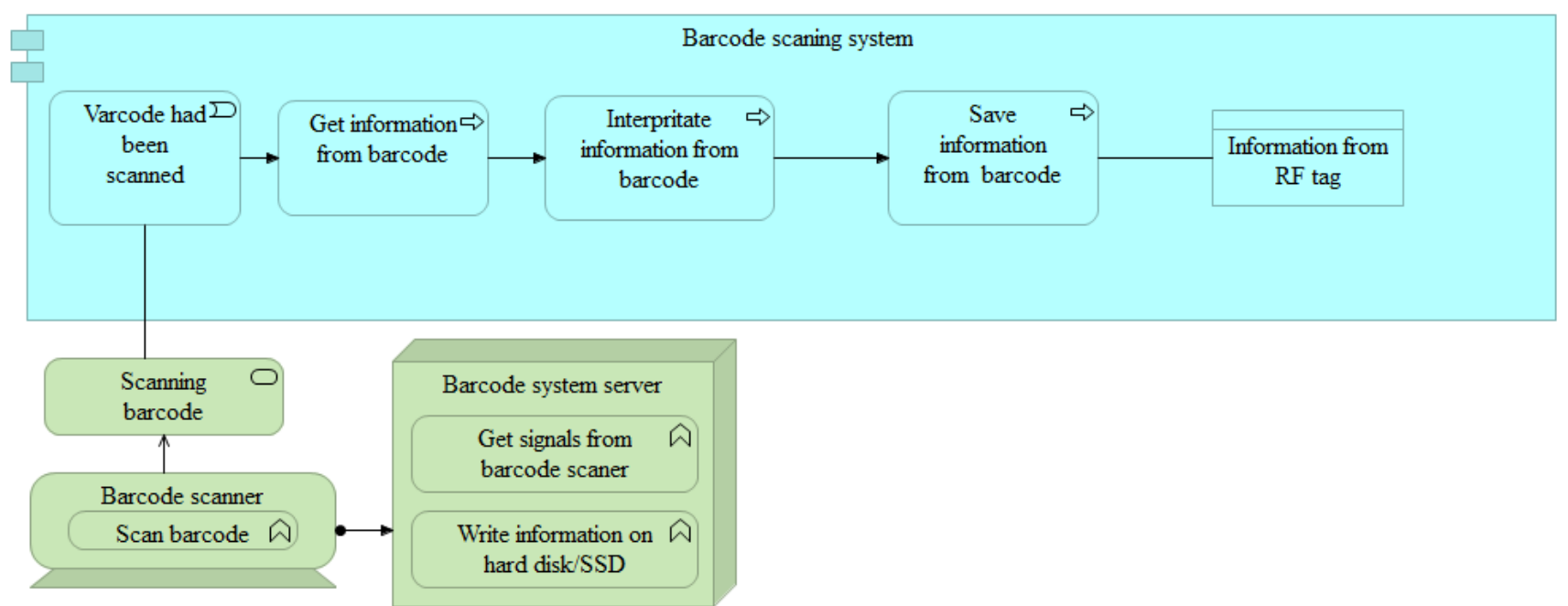

Figure 3 Barcode application scanning process

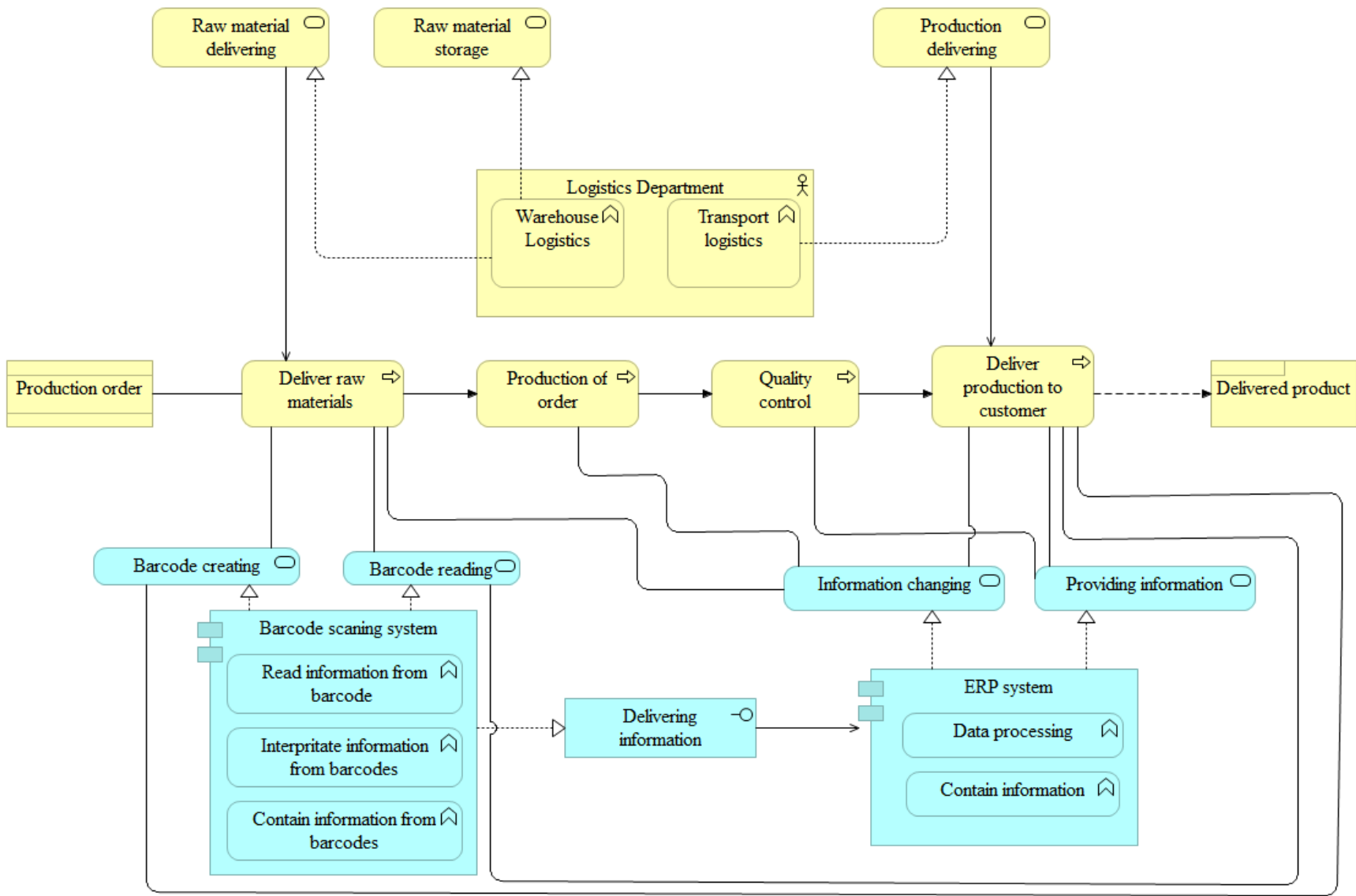

Figure 4 Producing order process to be.

You can see that of all the actions "print barcode" / "scan barcode", only the process of installing RF tags on the raw material remains. Consider how in this case a layer of applications will be transform (Fig. 6).
The main difference of the system using RFID technology is the automatic tag reading in the range of the reader. This reduces the number of routine operations, and also opens up the possibility of creating a heat map of the intensity of cells 
using and paths in the warehouse [20]. With this map, it is possible to optimize the location of resources in terms of the frequency of the resource using, cell and path. Depending on the frequency of the resource using, it can be placed in the cells located in the most passable areas, which will speed up the assembly time of the order. Adding sensors to RF readers will allow you to locate resources not only depending on the intensity of use, but also on storage conditions. Thus, the time for collecting resources will be reduced, the quality of storage of resources will increase. In addition to the heat map, you will be able to find out the following information about shipments:

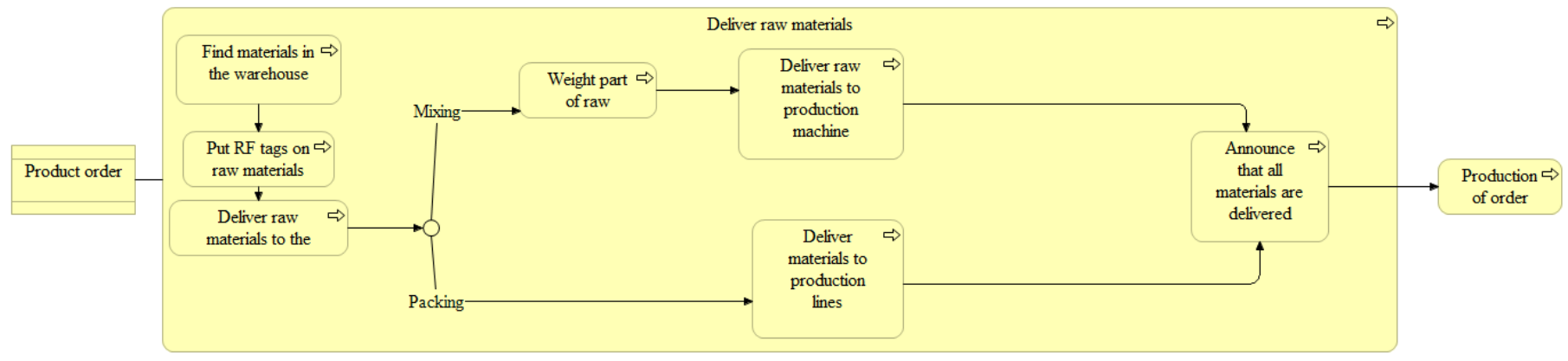

Figure 5 Decomposition of deliver raw materials process to be

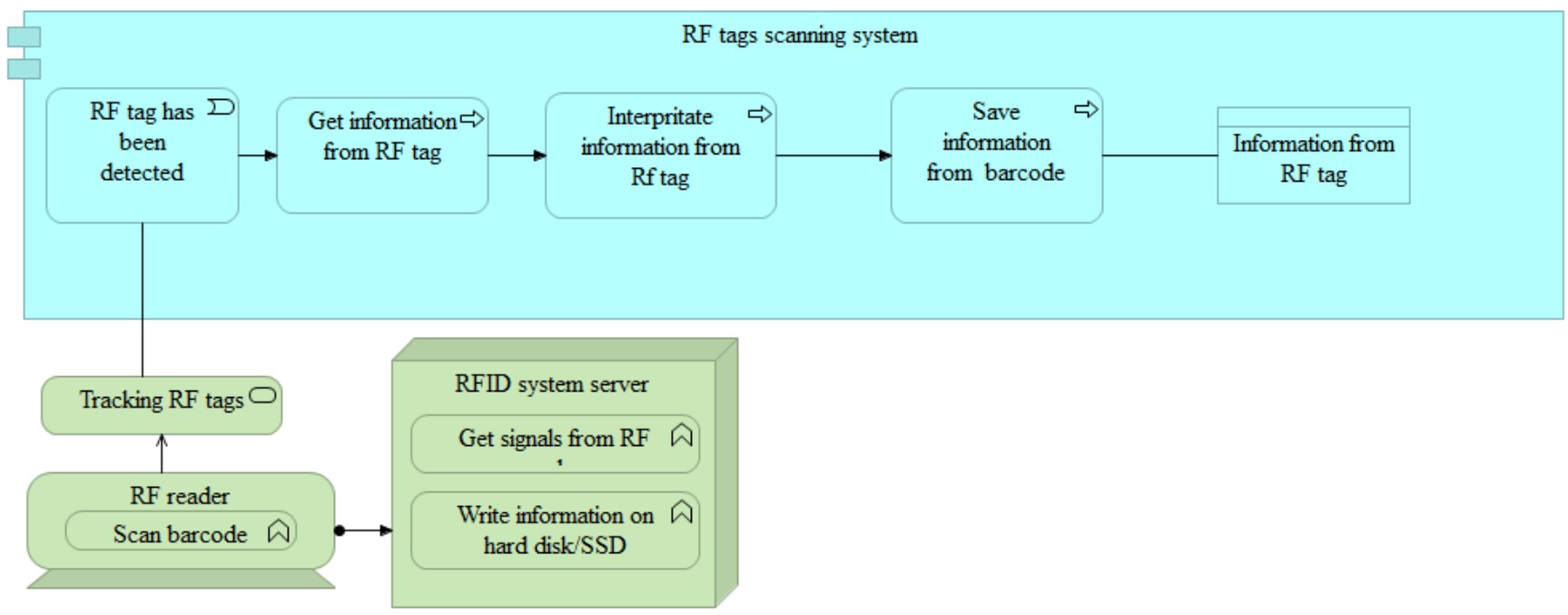

Figure 6 RF tag tracking application process

- in what temperature conditions this supply was stored;

- how often the delivery was used;

- how long the component is stored in the cell.

This information will also optimize the warehouse in terms of storage resources, as well as improve the quality control of the final product. Some of these data can be collected using the bar-coding system, however, due to problems with constant access to information stored in the barcode, the accuracy of such data will not be great, which will have a bad effect on the analysis of incoming information. Another factor is that RF tags can be reused. If the RFID is written incorrectly, the tags can be overwritten. In addition, labels are harder to damage than barcode stickers. Accordingly, the cost of consumables for printing will be reduced.

Another advantage of RF tags is the loyalty of technology to employee's errors. When using a bar-coding system, erroneously loaded packages must be scanned again, scanned the cart from which it is being moved, and the cell into which it is being moved. Then you need to take the necessary packaging, scan it, cell and trolley. That is, the number of actions with the scanner increases from 3 to 9 . When using RFID technologies, you need to put back the package, taken by mistake, and take the required one, then you need to overwrite the information on the RF tag. For one package, this difference may show insignificant, but given that dozens of components are necessary for one production, the time for their collection will be significantly reduced.

\section{CONCLUSION}

Summarizing, we can say that the use of RF tags reduces the time for collecting and delivering components, which will speed up the production of the order and respond more quickly to changes in the production plan.

The main advantage of the implementation of the RF system is the ability to move to a fully automated warehouse [21], which solves the problem of digital transformation of the company's logistics processes. The main problem of the barcode is the need for direct visibility. If for a person it is a task that is solved quickly enough, then for a robot it is almost impossible. The ability to read the RF tag, regardless of position, is the quality characteristic that will allow you to move to a fully automated warehouse in which a person plays 
a coordinating and controlling role. The robots themselves will be able to find the necessary component and deliver it to production, and, thanks to the data from the sensors and the heat map, the robots will understand at what point a particular component needs to be delivered. At the same time, robots will

\section{DISCUSSION}

Despite the advantages of RFID over bar-coding systems, it is necessary to clearly understand the purpose and feasibility of implementing this system. The main motivator of implementing a system based on RFID technology is the transition to a fully automated warehouse. Of course, at the intermediate stage, the use of RFID can also be beneficial, but it is incomparable with the cost of implementing such a system. This is due to the fact that the vast majority of the modern market uses barcode-based systems, as well as the high cost of the RFID system compared to bar-coding systems. However, every year the price of RF tags decreases (the price of a passive tag in 2019, on average, is 1 euro cent), and the technology itself is improving and becoming more accessible for wide consumer groups. Thus, the transition to RFID will be an integral part of the transition to a fully automated warehouse, which contributes to the digital transformation of the logistics processes of companies.

\section{ACKNOWLEDGMENT}

The reported study was funded by RSCF according to the research project № 19-18-00452.

\section{REFERENCES}

[1] Fabio De Felice, Antonella Petrillo, Stanislao Monfreda; Improving Operations Performance with World Class Manufacturing Technique: A Case in Automotive Industry; The open university of Hong Kong, 2018

[2] https://pubs.opengroup.org/architecture/archimate3-doc/toc.html

[3] Ilin, I., Levina, A., Abran, A., Iliashenko, O. (2017). Measurement of enterprise architecture (EA) from an IT perspective: Research gaps and measurement avenues. Paper presented at the ACM International Conference Proceeding Series, Part F131936, 232-243.

[4] Levina, A.I., Borremans, A.D., Burmistrov, A.N. Features of enterprise architecture designing of infrastructure-intensive companies (2018) Proceedings of the $31 \mathrm{st}$ International Business Information Management Association Conference, IBIMA 2018: Innovation Management and Education Excellence through Vision 2020, pp. 4643 4651 .

[5] Zaychenko, I.M., Ilin, I.V., Lyovina, A.I. Enterprise architecture as a means of digital transformation of mining enterprises in the Arctic (2018) pp. 4652-4659. perform these operations as quickly as possible, which is a step towards continuous digital production, which will provide the necessary advantage over competitors and increase the profitability of the enterprise.

[6] Anisiforov, A.B., Dubgorn, A.S. Organization of enterprise architecture information monitoring (2017) pp. 2920-2930.

[7] Roy Want, Intel Research; An Introduction to RFID Technology, Pervasive computing January-March 2006, pp. 25-33

[8] Amin, Emran Md; Karmakar, Nemai Chandra; Saha, Jhantu Kumar; Chipless RFID sensors, John Wiley \& Sons, 2016, ch 1.

[9] Alied market research; RFID Sensor Market by Product (Tags, Reader, and Software), Frequency Band (Low Frequency, High Frequency, and Ultrahigh Frequency) Type (Active and Passive), Application (Access Control, Livestock Tracking, Ticketing, Cashless Payment, and Inventory Management), and Industry Vertical (Transportation \& Logistics, Healthcare, Hospitality, Food \& Beverages, Retail, Manufacturing, and Government) - Global Opportunities Analysis and Industry Forecast, 2018-2024; 2018

[10] Maydanova, S., Ilin, I. Problems of the preliminary customs informing system and the introduction of the Single Window at the sea check points of the Russian Federation (2018) 239, 04004.

[11] Neil Farmer; Trends in packaging of food, beverages and other fastmoving consumer goods (FMCG): Markets, materials and technologies; Woodhead Publishing, 2013

[12] https://www.camcode.com/asset-tags/what-is-warehouse-logistics/

[13] Alesinskaya, T.V., Arutyunova, D.V., Orlova, V.G., Ilin, I.V., Shirokova, S.V. Conception BSC for investment support of port and industrial complexes (2017) Academy of Strategic Management Journal, 16 (Specialissue1), pp. 10-20.

[14] Lukashevich, N., Svirina, A., Garanin, D. Multilevel prognosis of logistics chains in case of uncertainty: Information and statistical technologies implementation (2018) 4 (1), 2.

[15] Doron Shaked, Avi Levy, Zachi Baharav, Jonathan Yen; A Visually Significant Two Dimensional Barcode; HP Laboratories Israel 2001

[16] Jihong Yu, Lin Chen; Tag Counting and Monitoring in Large-Scale RFID Systems; Springer International Publishing, 2019

[17] Pejvak Oghazi, Fakhreddin Fakhrai Rad, Stefan Karlsson and Darek Haftor; RFID and ERP systems in supply chain management; Emerald Publishing Limited, 2018

[18] Hamid Jabbar, Taikyeong Ted. Jeong; RFID System Integration; Intechopen, 2010

[19] John T. Yee, Seog-Chan Oh, Technology Integration to Business: Focusing on RFID, Interoperability, and Sustainability for Manufacturing, Logistics, and Supply Chain Managemen (2013) Springer-Verlag London ch 5-6

[20] Leland Wilkinson, Michael Friendly; The History of the Cluster Heat Map; The American Statistician, 2009

[21] https://www.industryweek.com/warehousing-anddistribution/automation-warehouse-asset-or-obstacle 